\title{
A Novel Classification Approach Capable Indoor Scene Picture Identification with Hybrid Feature Selection Algorithm
}

\author{
GaganDeep Singh \\ Research Scholar, Department of Computer Science \\ \& Engineering \\ Shaheed Bhagat Singh State Technical Campus, \\ Ferozepur (Punjab), India
}

\author{
Sonika Jindal \\ Department of Computer Science and Engineering \\ Shaheed Bhagat Singh State Technical Campus \\ Ferozepur, India
}

\begin{abstract}
Image scene classification is an integral part of several aspects of image process. Indoor and outside classification could be a elementary part of scene process because it is that the place to begin of the many linguistics scene analysis approaches. Several novel techniques are developed to tackle this drawback, however every technique depends on its own information of pictures therefore reducing the boldness within the success of every technique. The planned model is formed capable of operating with the variations within the indoor scene image dataset, that are noticed within the sort of the color, texture, light, image orientation, occlusion and color illuminations. many experiments has been conducted over the projected model for the performance analysis of the indoor scene recognition system within the planned model. The results of the proposed model are obtained in the type of the various performance parameters of applied mathematics errors, precision, recall, F1-measure and overall accuracy. The planned technique has clearly outperformed the present models within the terms of the accuracy. The planned model improvement has been recorded above ten percent for all of the evaluated parameters against the prevailing models based mostly upon SURF, FREAK, etc.
\end{abstract}

\section{General Terms}

Digital Image Processing, Classification, Pattern Recognition, Feature Extraction

\section{Keywords}

Multi-class SVM, Classification, Feature Selection, Indoor Scene Identification, SURF

\section{INTRODUCTION}

Scene classification is aimed toward labeling a picture into linguistics classes (room, office, mountain etc.). It's a very important task to classify, organize and perceive thousands of pictures with efficiency. From application purpose of view, scene classification is beneficial in content based mostly image retrieval. As correct classification of a picture, is good because it helps in higher organization and browsing of the image information. Scene classification is very valuable in remote navigation additionally [1].

Indoor scenes are littered with several objects. Thus classification techniques merely supported color; texture and intensity don't seem to be terribly effective to classify indoor scenes. Pioneering works used SIFT, SURF etc. together with supervised learning. However these techniques fail to differentiate several indoor scenes. a way to bridge linguistics gap between image illustration and image recognition is to create use of more and more refined models, however smart learning and reasoning is very troublesome task for such models. as an alternative linguistics gap between low-level options like color, intensity, texture etc. and introducing object-based illustration as intermediate illustration will cut back high-level class label. Because the performance of scene recognition is [5] heavily obsessed with feature illustration, this object-based intermediate illustration proves to be helpful in enhancing classification results. Recently objects-based techniques for indoor scene classification have verified to be showing promising performance over alternative state-of-art techniques.

\subsection{Classifier}

Support vector machines were planned by Boser et al. in [9]. SVM is supervised machine learning approach specifically designed for pattern matching. SVMs construct a group of hyper-planes that separates the info points into 2 categories with supreme margin in high dimensional feature area. Mathematically, SVM learns a mapping $\chi \rightarrow \Upsilon$ wherever $\mathrm{x} € \chi$ represents the feature vector and $\mathrm{y} € \Upsilon$ represents scene class.

\section{LITERATURE REVIEW}

Monadjemi, Amir et. al.[2] have performed the experiments on high resolution pictures towards outside scene classification. Authors have examined the utilization of high frequency options in high-resolution pictures to extend texture classification accuracy once utilized in combination with lower frequency options. They used Gabor options derived from sections of 4032 nine 2688 pictures. A neural network classifier was wont to confirm the classification performance of lower and high frequency options once used one by one then together. Feature shuffling and Principal element Analysis was applied to work out each the role of every feature within the classification and to extract a smaller reduced feature set involving each lower and high frequency options. Duda, Richard O., Peter E. Hart, and David G. Stork [3] have worked on pattern classification for robotic vision based mostly scene classification. The authors have given the essential results and definitions from algebra, applied math, scientific theory and procedure quality that function the mathematical foundations for the pattern recognition techniques mentioned throughout this book. They need tried to grant intuition whenever acceptable, however we tend to don't arrange to prove these results; systematic expositions will be found within the references. Fitzpatrick, Paul [4] has developed an Indoor/outdoor scene classification project. Authors have powerfully related to with classes we tend to might imagine treating statistically. He has replicated the human labeling of inexperienced and sky, the information suggests we tend to might attain success rates of around eighty-six. Detection folks would appear slightly additional di_cult, however this dimension has indeed very little power 
for discrimination anyway, since folks tend to look in any photograph in spite of class. Quattoni et al. [5] have remarked that actual state of the art algorithms failed on recognizing indoor scenes due to large variability across different exemplars within each class. They have presented an approach to use prototypes to detect regions of interest (ROIs) for recognizing indoor scenes. Each prototype consisted of manually annotated ROIs for each prototype. Small spatial windows were used for searching each ROI in image. They have introduced a large dataset containing wide range of input categories i.e. 67 categories, which is used as benchmark for indoor scene classification dataset. Li-Jia $\mathrm{Li}$ et al. [6] have collectively worked upon to analyze the contribution of objects to scene classification. This paper discusses the utility of image illustration supported objects in providing complementary info to the low-level options. During this work an outsized variety of 'object filters' are used. They need used object-bank of two hundred pre-trained SVM object detector at twelve totally different detection scales and three abstraction pyramid levels. Object bank illustration yields promising results by capturing necessary info. However, limitation of this lies in computation speed and high spatiality of object-bank. The coaching of an outsized variety of object detectors is additionally troublesome. They any increased performance by commutation SVM classifier with supplying regression classifier. They explore feature sparsely and object sparsely for spatiality reduction.

\section{EXPERIMENTAL DESIGN}

Indoor scene recognition involves varied steps like feature extraction, feature choice, feature vector generation, train the system and classification.

\subsection{Feature Extraction}

The following is that the feature matching and classification formula for matching the extracted indoor scene image with the various pictures of same scene, that are taken at totally different times, from totally different viewpoints, or by totally different sensors.

\section{Algorithm 1: Feature Extraction Algorithm}

1. Load the 3-D (colored) test image as test object matrix $\mathbf{T}_{\mathbf{m}}$

2. Convert the test image matrix to grayscale image matrix $\mathbf{G}_{\mathbf{m}}$

3. Define the Gaussian Filter Gf

4. Apply Gaussian Filter $\mathbf{G} \mathbf{f}$ on $\mathbf{G}_{\mathbf{m}}$ to produce the denoised $\mathbf{G}_{\mathbf{m d}}$

5. Calculate $\mathbf{G}_{\mathbf{m d}}$ into the front-ground estimation feature $\mathbf{F}_{\mathbf{E F}}$

6. Define the dilation object of adequate shape and size $\mathbf{S}_{\mathbf{E} 1}$

7. Dilate the image $\mathbf{G}_{\mathbf{m d}}$ with respect to $\mathbf{S}_{\mathbf{E} \mathbf{1}}$ to produce the image object $\mathbf{A}_{\mathbf{I}}$

8. Perform morphological closing of the image object $\mathbf{A}_{\mathrm{I}}$

9. Subtract the $\mathbf{F}_{\mathbf{E F}}$ from $\mathbf{G}_{\mathbf{m}}$ to produce $\mathbf{F}(\mathbf{B G})$

10. Return the $\mathbf{F}(\mathbf{B G})$
Algorithm 2: Classification with Appearance based feature descriptor

1. Read the source image, and Extract the features from the source indoor scene image. Feature descriptor will be the sub image, and will describe smaller details than the original Target image.

2. Perform pre-processing step to validate the feature descriptor set and prepare all of the feature descriptors within the single feature sets because the training set.

3. Prepare the cluster data by adding the cluster IDs corresponding with all of the samples or feature descriptors within the coaching set.

4. Run SVM coaching on the feature descriptor coaching set and come back the load and bias data for all feature descriptors within the coaching set.

5. Run SVM classifier by submitting the SVM weight and bias knowledge, cluster data and therefore the testing feature descriptor vector.

6. Come back the matching SVM classification data.

7. Appraise the SVM classification data and come back the choice logic.

\subsection{Simulation Environment}

The details of implementation of the proposed model have been discussed under this chapter. Firstly, the proposed model has been developed using the MATLAB simulator. The results have been obtained from various aspects.

\section{RESULTS}

\subsection{Performance Parameters}

The performance of the proposed indoor scene recognition model has been verified under this chapter. The section explains the performance parameters utilized for the purpose of evaluation of the results proposed model.

Table 1: Hypothesis decision parameter entities in type 1 and 2 errors

\begin{tabular}{|l|l|l|l|}
\hline & $\begin{array}{l}\text { Doesn't Have The } \\
\text { Condition } \\
\text { (Satisfies } \\
\text { Hypothesis N Null }\end{array}$ & $\begin{array}{l}\text { Has } \\
\text { Condition } \\
\text { (Does Not Satisfy } \\
\text { Null Hypothesis }\end{array}$ \\
\hline $\begin{array}{l}\text { Tests Negative } \\
\text { (Null Accepted) }\end{array}$ & $\begin{array}{l}\text { True Negative } \\
\text { TN or } \mathrm{n}_{00}\end{array}$ & $\begin{array}{l}\text { False Negative } \\
\text { FN or } \mathrm{n}_{10}\end{array}$ \\
\hline $\begin{array}{l}\text { Tests Positive } \\
\text { (Null Rejected) }\end{array}$ & $\begin{array}{l}\text { False } \\
\text { FP or } \mathrm{n}_{01}\end{array} \quad$ Positive & $\begin{array}{l}\text { True Positive } \\
\text { TP or } \mathrm{n}_{1}\end{array}$ \\
\hline
\end{tabular}

The statistical parameters to measure the statistical errors (Typeface 1 and Typeface 2) are measured in order to evaluate the overall performance of the proposed model by evaluating the samples by the means of the programming or the manual binary classification. The proposed model evaluation is entirely based upon this statistical analysis. Table 1 explains the significance of the typeface 1 and typeface 2 statistical errors for the evaluation of the hypothesis.

\subsection{Analysis with 100 test cases}

The first experiment has been conducted over the 100 check samples that are randomly elect out of the given image set. The randomizer module generates the random index containing the hundred image ids that are not any inherited from the given dataset. Such at random chosen samples are a 
lot of processed and analyzed below the planned model for the result analysis.

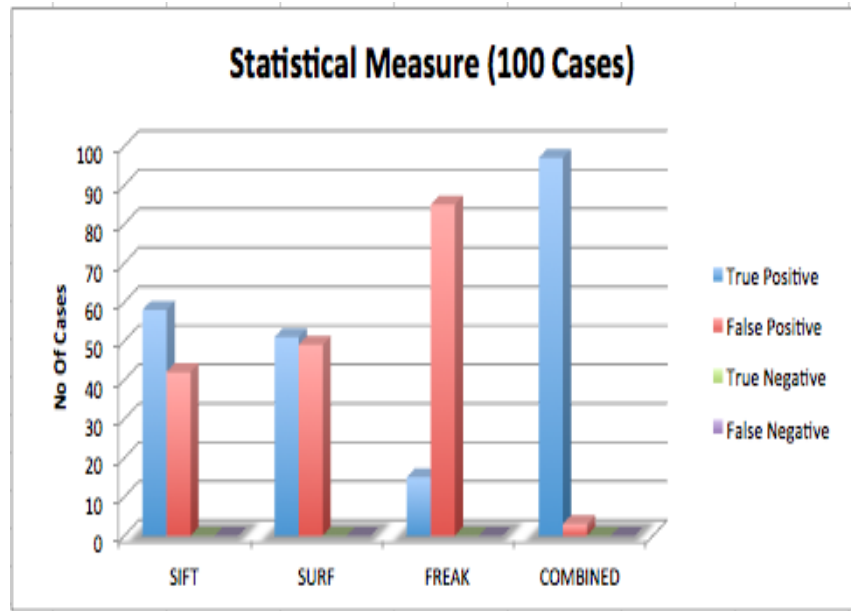

Fig 1: Typeface1 and typeface 2 errors for 100 test cases

The typeface one and typeface two errors has been evaluated from the checking of the input check samples. The graph obtained from the values of the mathematics kind one and a combine of errors has been given among the figure1. The figure1 has been obtained from the hundred testing samples and each one in every of the samples show the equal mathematics errors from the first. Obtained Values has been shown in Table 2.

Table 2: Error Rate for Outcomes

\begin{tabular}{|l|l|l|l|l|}
\hline & SIFT & SURF & FREAK & COMBINED \\
\hline True Positive & 58 & 51 & 15 & 97 \\
\hline False Positive & 42 & 49 & 85 & 3 \\
\hline True Negative & 0 & 0 & 0 & 0 \\
\hline False Negative & 0 & 0 & 0 & 0 \\
\hline
\end{tabular}

Table 2 defines the values for the Figure 1. Table 2 contains the statistical errors obtained from the a hundred randomly chosen samples.

Figure 2 shows the implementation measures computed over the obtained one hundred input samples.

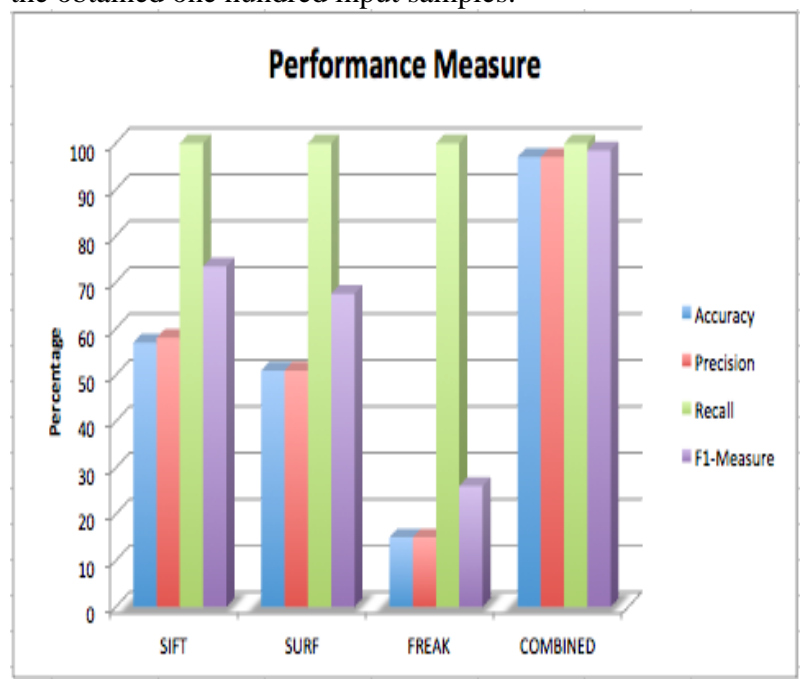

Fig 2: Performance measures for 100 Input Samples
The designed technique and alternative models supported SIFT, SURF and FREAK has been recorded with the flexible performance measure over the given applied math errors.

Table 3: Performance measures for 100 test cases

\begin{tabular}{|l|l|l|l|l|}
\hline & SIFT & SURF & FREAK & COMBINED \\
\hline Accuracy & 57 & 51 & 15 & 97 \\
\hline Precision & 58 & 51 & 15 & 97 \\
\hline Recall & 100 & 100 & 100 & 100 \\
\hline $\begin{array}{l}\text { F1- } \\
\text { Measure }\end{array}$ & 73.417 & 67.54967 & 26.08696 & 98.477 \\
\hline
\end{tabular}

\section{CONCLUSION}

The projected model has been planned on the evidence of the various feature selectors for the indoor scene recognition. The image feature abstraction strategies play the most important role within the indoor scene recognition by extracting the helpful options that are estimated to investigate the class of the input query picture. The low-level feature evaluator decreases the pictorial belongings of the pictures that make it easier to search out the tougher areas among the image data. The stronger regions are inter-matched by using the wellorganized classification methodology so as to assess the indoor scene recognition within the given image data set. During this thesis, the combined approach of SIFT, SURF and FREAK along side the multi-class support vector machine (mSVM) has been projected for the indoor scene recognition. The probabilistic categorization with the multi-class support vector machine has been developed for the hardiness within the categorization. The planned model has experienced many experiments and has been originate higher than the previous possibility within the terms of precision, recall, f1-measure and overall accuracy and Combined model has outperformed the other descriptors with mSVM classification.

\subsection{Future Work}

In the future the proposed model can be improved by using the hybrid low level feature extracted along with the efficient color illumination to find the dual-mode attacks over the images to determine the indoor scene in the image data. Also the swarm intelligent algorithm can be utilized for the indoor scene recognition in the digital image dataset

\section{REFERENCES}

[1] $\mathrm{Li}$, Yansheng, et al. "Unsupervised multilayer feature learning for satellite image scene classification." IEEE Geoscience and Remote Sensing Letters 13.2 (2016): 157-161.

[2] Monadjemi, Amir, B. T. Thomas, and Majid Mirmehdi. Experiments on high resolution images towards outdoor scene classification. Technical report, University of Bristol, Department of Computer Science, 2002.

[3] Duda, Richard O., Peter E. Hart, and David G. Stork. Pattern classification. John Wiley \& Sons,, 1999.

[4] Fitzpatrick, Paul. "Indoor/outdoor scene classification project." Pattern Recognition and Analysis. 
[5] Quattoni, Ariadna, and Antonio Torralba. "Recognizing indoor scenes." InComputer Vision and Pattern Recognition, 2009. CVPR 2009. IEEE Conference on, pp. 413-420. IEEE, 2009.

[6] Li, Li-Jia, Hao Su, Yongwhan Lim, and Li Fei-Fei. "Objects as attributes for scene classification." In Trends and Topics in Computer Vision, pp. 57-69. Springer Berlin Heidelberg, 2012.

[7] Antanas, Laura, Marco Hoffmann, Paolo Frasconi, Tinne Tuytelaars, and Luc De Raedt. "A relational kernel-based approach to scene classification." InApplications of Computer Vision (WACV), 2013 IEEE Workshop on, pp. 133-139. IEEE, 2013.

[8] Mesnil, Grégoire, Salah Rifai, Antoine Bordes, Xavier Glorot, Yoshua Bengio, and Pascal Vincent. "Unsupervised and Transfer Learning under Uncertainty-
From Object Detections to Scene Categorization." In ICPRAM, pp. 345-354. 2013

[9] Zhang, Lei, Xiantong Zhen, and Ling Shao. "Learning object-to-class kernels for scene classification." Image Processing, IEEE Transactions on 23, no. 8 (2014): 3241-3253.

[10] Li, Li-Jia, Hao Su, Li Fei-Fei, and Eric P. Xing. "Object bank: A high-level image representation for scene classification \& semantic feature sparsification." In Advances in neural information processing systems, pp. 1378-1386. 2010.

[11] Alberti, Marina, John Folkesson, and Patric Jensfelt. "Relational approaches for joint object classification and scene similarity measurement in indoor environments." In AAAI 2014 Spring Symposia: Qualitative Representations for Robots.2014. 